\title{
Sex Disparities in Productivity among Oculoplastic Surgeons
}

\author{
Mckenzee Chiam, BS ${ }^{1}$ Mona L. Camacci, MD, MS ${ }^{2}$ Alicia Khan, BS 3 Erik B. Lehman, MS
}

Seth M. Pantanelli, MD, MS ${ }^{1}$

${ }^{1}$ Department of Ophthalmology, Penn State College of Medicine, Hershey, Pennsylvania

2 Department of Ophthalmology, David \& Ilene Flaum Eye Institute University of Rochester, Rochester, New York

${ }^{3}$ School of Medicine, Georgetown University, Washington, Dist. of Columbia

${ }^{4}$ Department of Public Health Sciences, Penn State College of

Medicine, Hershey, Pennsylvania

\begin{abstract}
Address for correspondence Seth M. Pantanelli, MD, Department of Ophthalmology, Penn State College of Medicine, 500 University Drive, HU19, Hershey, PA 17033-085 (e-mail: spantanelli@pennstatehealth.psu.edu).
\end{abstract}

J Acad Ophthalmol 2021;13:e210-e215.

\begin{abstract}
Keywords

- oculoplastic

- ophthalmology

- academic rank

- publication productivity

- NIH funding

- disparities

- gender

Purpose The aim of the study is to investigate sex differences in academic rank, publication productivity, and National Institute of Health (NIH) funding among oculoplastic surgeons and whether there is an association between American Society of Ophthalmic Plastic and Reconstructive Surgery (ASOPRS) membership and scholarly output.

Methods Sex, residency graduation year, and academic rank were obtained from institutional websites of 113 U.S. ophthalmology programs. H-indices and m-quotients were obtained from the Scopus database. NIH funding information was obtained from the NIH Research Portfolio Online Reporting Tool.

Results Of the 272 surgeons, 74 (30.2\%) were females. When adjusted for career duration, differences in female to male proportions were only significant at the rank of assistant professor (assistant: 74.3 vs. $48.5 \%, p=0.047$; associate: 18.9 vs. $24.6 \%$, $p=0.243$; full professor: 13.0 vs. $37.2 \%, p=0.114$ ). Women had a shorter career duration than men [10.0 (interquartile range or IQR 12.0) vs. 21.0 (IQR 20.0) years; $p<0.001$ ] and a lower $h$-index [4.0 (IQR 5.0) vs. 7.0 (IQR 10.0); $p<0.001]$, but similar $m$ quotients [0.4 (IQR 0.4) vs. 0.4 (IQR 0.4); $p=0.9890]$. Among ASOPRS members, females had a lower $h$-index than males [5.0 (IQR 6.0) vs. 9.0 (IQR 10.0); $p<0.001$ ] due to career length differences. No difference in productivity between sexes was found among non-ASOPRS members. ASOPRS members from both sexes had higher scholarly output than their non-ASOPRS counterparts. Just $2.7 \%(2 / 74)$ of females compared with $5.3 \%(9 / 171)$ of males received $\mathrm{NIH}$ funding $(p=0.681)$.

Conclusion Sex differences in academic ranks and $h$-indices are likely due to the smaller proportion of females with long career durations. ASOPRS membership may confer opportunities for increased scholarly output.
\end{abstract}

received

May 16, 2021

accepted after revision

September 13, 2021
DOI https://doi.org/ $10.1055 / \mathrm{s}-0041-1740312$. ISSN 2475-4757.

\footnotetext{
(C) 2021. The Author(s).

This is an open access article published by Thieme under the terms of the Creative Commons Attribution-NonDerivative-NonCommercial-License, permitting copying and reproduction so long as the original work is given appropriate credit. Contents may not be used for commercial purposes, or adapted, remixed, transformed or built upon. (https://creativecommons.org/ licenses/by-nc-nd/4.0/)

Thieme Medical Publishers, Inc., 333 Seventh Avenue, 18th Floor, New York, NY 10001, USA
} 
Women represent a quarter (25.3\%) of all ophthalmologists and roughly $22.5 \%$ of oculoplastic surgeons. ${ }^{1,2}$ As with all fields in medicine, the proportion of females within ophthalmology is expected to increase as women comprise roughly half of U.S. medical graduates (48\%) and ophthalmology residents (44.3\%)., Although more women are entering the field of ophthalmology, previous studies have demonstrated that females continue to be under-represented in leadership positions both nationally and within their institutions. ${ }^{5,6}$ Several previous studies have analyzed female ophthalmologists within academic institutions, finding that women make up only $10 \%$ of department chairs; likewise, $13 \%$ of females compared with $34 \%$ of males achieve the rank of full professor. ${ }^{7,8}$

One plausible explanation for the sex differences seen in advanced ranks may be related to scholarly output and National Institute of Health (NIH) funding. The $h$-index has been used as a reliable tool to assess publication productivity in many fields. ${ }^{9}$ It is also positively correlated with increasing academic rank. ${ }^{10} \mathrm{~A}$ prior study evaluating ophthalmologists noted lower $h$-indices among females during earlier stages of their careers. ${ }^{8}$ However, one of the faults of the $h$-index is that it fails to account for an author's career duration, which is an important factor when analyzing groups with varying career durations, such as female versus male ophthalmologists. The $m$-quotient is a tool that calculates publication productivity while adjusting for an individual's career duration. ${ }^{9}$ Presence of NIH funding among ophthalmologists is also positively correlated with publication productivity. ${ }^{11}$ However, previous studies have shown disparities in NIH funding among ophthalmologists with female principal investigators (PIs) receiving lower award amounts compared with their male counterparts. ${ }^{12}$

Although previous studies have compared the publication productivity of oculoplastic surgeons to other subspecialties, the studies were limited; in that none investigated sex differences within the field of oculoplastic surgery. ${ }^{13}$ Many oculoplastic surgeons are also a member of a highly regarded organization, the American Society of Ophthalmic Plastic and Reconstructive Surgery (ASOPRS), which promotes scientific research involvement among its members. The purpose of this study was to investigate sex differences among academic oculoplastic surgeons in terms of academic rank, scholarly productivity, and $\mathrm{NIH}$ funding, while also determining the role of ASOPRS membership in publication productivity.

\section{Materials and Methods}

This cross-sectional study was deemed non-human research by the Institutional Review Board of the Penn State College of Medicine; therefore, informed consent was not required. A search of all ophthalmology residency-training programs participating in the 2019 San Francisco match yielded a total of 114 programs. One program was excluded due to lack of a faculty roster on the institutional website and information was not attainable through direct email communication, resulting in 113 programs included in the final analysis.
Official institutional websites were accessed between January and March 2019 to obtain a list of all the oculoplastic faculty at each institution. Using official institutional websites and search engines, data on sex, year of residency graduation, and academic rank on each oculoplastic surgeon were gathered. The sex of each individual faculty was confirmed using photographs, pronouns, profiles, and other supporting online search tools. Career duration of each individual faculty was calculated based on the number of years between their graduation year from residency and the year 2019, which was the year of this study's data acquisition. Residency instead of fellowship graduation year was collected because residency graduation year was more easily accessible.

The Scopus database (Elsevier, https://www.scopus.com) was used to determine each faculty member's total number of publications, number of citations on those papers, and the $h$-index. Attempts were made to determine any alternative names that faculty members may have had via online searches of curricula vitae and Scopus website profiles to capture publications under previously used names. The $h$ index, a measure of the scholarly productivity and the citation impact of an author, is calculated based on the highest number of publications an author has received with at least the same number of citations. ${ }^{9}$ M-quotient, which adjusts for varying career durations, was calculated by dividing the $h$-index by the author's career duration. ${ }^{9}$

The NIH Research Portfolio Online Reporting Tool Expenditures and Results (RePORTER) (https://report.nih.gov) was used to gather data on total dollar amount of $\mathrm{NIH}$ funding and number of projects funded for each oculoplastic surgeon. All faculty names and alternative names were queried. This database captures $\mathrm{NIH}$ funding from the years 1985 to present. Available data for each faculty member for all available years was included for analysis.

Data was managed using Research Electronic Data Capture (REDCap) and statistical analyses were performed using SAS version 9.4 (SAS Institute, Cary, NC). ${ }^{14}$ All $p$-values $<0.05$ were considered significant. All median data were reported with interquartile ranges (IQR). A binomial logistic regression model was conducted to compare academic ranks as binary response variables in terms of percentages between sexes, while adjusting for career duration ( - Table 1 ). Wilcoxon Rank Sum tests and medians were used to compare career duration between sexes at each academic rank, and productivity metrics including the median $h$-indices and $m$ quotients, between sexes. The same comparison was performed at three career duration intervals $(0-15,16-28$, and $\geq 29$ years) and at each academic rank (assistant, associate, and full professorship). Quantile regression models adjusted for career duration were conducted to compare median total number of publications, citations, $h$-indices, $m$-quotients, and $\mathrm{NIH}$ funding between sexes.

\section{Results}

A total of 272 oculoplastic surgeons were identified across 113 institutional websites. Oculoplastic surgeons with 
e212 Sex Disparities among Oculoplastic Surgeons Chiam et al.

Table 1 Academic oculoplastic surgeons based on sex and academic rank

\begin{tabular}{|c|c|c|c|}
\hline Academic rank & Female & Male & $p$-Value \\
\hline \multicolumn{4}{|l|}{ Assistant Professor } \\
\hline Percentage $(n)$ & $74.3 \%(55 / 74)$ & $48.5 \%(83 / 171)$ & $0.047^{b}$ \\
\hline Median career duration (IQR) ${ }^{\mathrm{a}}$ & $9.0(7.0)$ & $13.5(19.0)$ & $<0.001^{\mathrm{c}}$ \\
\hline \multicolumn{4}{|l|}{ Associate Professor } \\
\hline Percentage $(n)$ & $18.9 \%(14 / 74)$ & $24.6 \%(42 / 171)$ & 0.243 \\
\hline Median career duration (IQR) ${ }^{a}$ & $15.5(11.0)$ & $15.0(13.0)$ & $0.894^{c}$ \\
\hline \multicolumn{4}{|l|}{ Full Professor } \\
\hline Percentage $(n)$ & $6.8 \%(5 / 74)$ & $26.9 \%(46 / 171)$ & $0.114^{\mathrm{b}}$ \\
\hline Median career duration (IQR) ${ }^{\mathrm{a}}$ & $24.0(10.0)$ & $33.0(10.0)$ & $0.136^{c}$ \\
\hline \multicolumn{4}{|l|}{ Overall } \\
\hline Percentage $(n)$ & $30.2 \%(74)$ & $69.8 \%(171)$ & \multirow[t]{2}{*}{$<0.001^{c}$} \\
\hline Median career duration (IQR) & $10.0(12.0)$ & $21.0(20.0)$ & \\
\hline
\end{tabular}

${ }^{a}$ Median career duration shown in years with interquartile range (IQR).

${ }^{b} p$-Value from binomial logistic regression model adjusted for career duration.

${ }^{c} p$-Value from Wilcoxon Rank Sum test with significant results $(p<0.05)$ bolded.

insufficient online information to complete all biographic data collection $(n=25)$ or faculty listed as part time $(n=2)$ were excluded from the analysis, yielding a total sample of 245 oculoplastic faculty. Of these faculty members, 74 (30.2\%) were female and 171 (69.8\%) were male. In addition to holding an MD or a DO, female and male oculoplastic surgeons also held additional $\mathrm{PhD}[1$ (1.4\%) vs. 9 (5.3\%); $p=0.205$ ], MS [ 1 (1.4\%) vs. 6 (3.5\%); $p=0.242$ ], MPH [1 (1.4\%) vs. $8(0.6 \%) ; p=0.782$ ], and other degrees [1 (3.1\%) vs. 5 (2.9\%); $p=0.701]$.

\section{Sex Differences within Academic Institutions}

When analyzing academic rank without adjusting for career duration, a greater proportion of females [74.3\% (55/74)] vs. males [48.5\% (83/171)] were assistant professors $(p=0.002)$, and a smaller proportion of females [6.8\% (5/74)] compared with males [26.9\% (46/171)] were full professors $(p=0.004)$. Similar proportions of females and males were associate professors [18.9\% (14/74) vs. 24.6\% (42/171); $p=0.243$ ]. However, when adjusting for career duration, the statistical significance between proportions of females and males at each academic rank attenuated and only a significant difference among assistant professors remained (-Table 1). Overall, female oculoplastic surgeons had a significantly shorter median career duration compared with their male colleagues ( - Table 1). The career duration distribution of females and males are summarized in - Fig. 1. When evaluated by academic rank, females had shorter median career durations than males at all three ranks, but this difference was only statistically significant at the assistant professor level (-Table 1).

Oculoplastic surgeons comprised 7.1\% (8/113) of all residency program directors, of whom $1.8 \%(2 / 113)$ were female and $5.3 \%(6 / 113)$ were male $(p=0.533)$. Oculoplastic surgeons also represented $7.1 \%$ of all department chairs (8/113), of whom all were male; they had a median career duration of 30.5 years.

\section{Sex Differences in Bibliometrics}

Bibliometric differences were seen in female and male oculoplastic surgeons. Females versus males had a lower median number of publications [10.0 (IQR 22.0) vs. 23.0 (IQR 56.0); $p=0.200$ ] and lower total number of citations [90.0 (IQR 263.0 ) vs. 188.0 (IQR 654.0); $p=0.184$ ], although these findings were not statistically significant in the quantile regression models that adjusted for career duration. Additionally, when looking at citations received per year, females had similar median number of publications per year compared with males [1.3 (IQR 2.1) vs. 1.4 (IQR 2.9); $p=0.169$ ] and similar median citations received per year [8.3 (IQR 24.1) vs. 11.4 (IQR 28.4); $p=0.335$ ].

Females had a lower overall median $h$-index compared with males [4.0 (IQR 5.0) vs. 7.0 (IQR 10.0); $p=0.009$ ], but no significant difference was found in median m-quotients

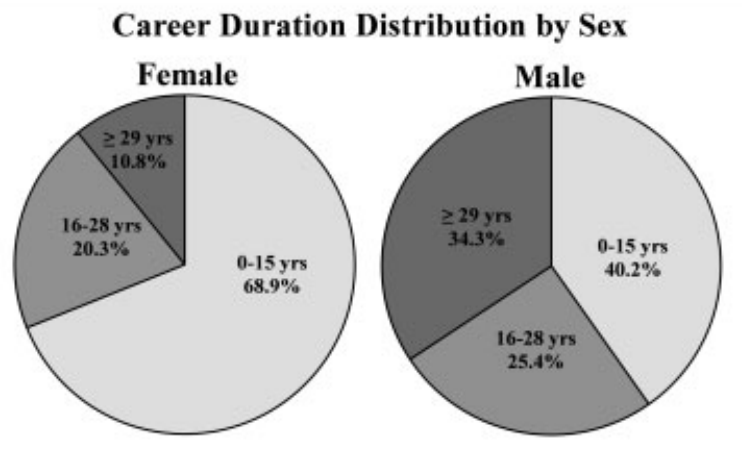

Fig. 1 Career duration distribution of oculoplastic surgeons by sex. Career duration intervals calculated based on the number of years since residency graduation. 
A

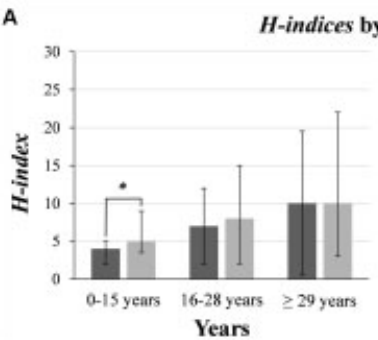

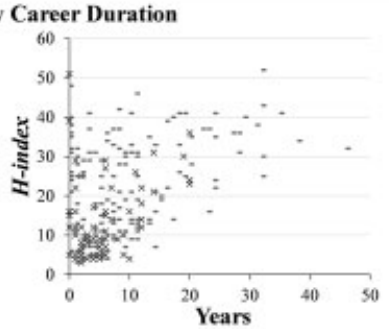

M-quotients by Career Duration

B
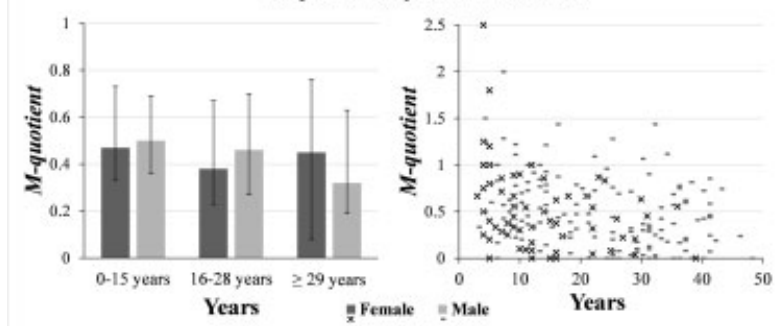

Fig. 2 Bibliometrics for oculoplastic surgeons by career duration and sex. (A) $\mathrm{H}$-indices by career duration. Significant difference in h-index between sexes was found at the 0 to 15 years subgroup $(p=0.005)$. (B) M-quotients by career duration. Median values with error bars representing interquartile ranges shown on bar graphs (left). Scatter plots showing absolute indices for each individual surgeon (right). ${ }^{*} p$ $<0.05$.

between sexes [0.4 (IQR 0.6) vs. 0.4 (IQR 0.5); $p=1.00$ ]. When analyzed by career duration interval, there was a significant difference in $h$-indices between females and males among surgeons with career durations of 0 to 15 years $(p=0.005)$, but no significant difference was seen at other career duration intervals (-Fig. 2). When evaluated by academic rank, the $h$ indices and $m$-quotients were equivalent for females and males (-Fig.3).The $h$-index and $m$-quotient of each individual female or male surgeon, plotted against the number of years in practice, are illustrated in scatter plots in $\mathbf{- F i g . ~} \mathbf{2}$.

\section{Sex Differences and ASOPRS Membership}

A total of 166 (67.8\%) academic oculoplastic surgeons were identified to be a member of the ASOPRS, of whom 47 (28.3\%) were female and 119 (71.7\%) were male. Among ASOPRS members, females had a significantly lower median $h$-index than males [5.0 (IQR 6.0) vs. 9.0 (IQR 10.0); $p<0.001$ ], but had similar median m-quotients [0.5 (IQR 0.4) vs. 0.5 (IQR $0.4) ; p=0.925]$. Among non-ASOPRS members, females and males had both similar median $h$-indices [2.0 (IQR 4.0) vs. 3.0 (IQR 4.0); $p=0.909]$ and $m$-quotients [0.3 (IQR 0.4) vs. 0.3 (IQR 0.3); $p=0.597$ ] as shown in - Fig. 4. Females who were members of ASOPRS had a higher median $h$-index [5.0 (IQR 6.0 ) vs. 2.0 (IQR 4.0 ); $p=0.004$ ], but similar m-quotients [0.5 (IQR 0.4 ) vs. 0.3 (IQR 0.4 ); $p=0.078$ ] than non-ASOPRS females. Males who were members of ASOPRS had a higher median $h$-index [9.0 (IQR 10.0) vs. 3.0 (IQR 4.0); $p<0.001$ ] and a higher median m-quotient [0.5 (IQR 0.4 ) vs. 0.3 (IQR $0.3) ; p<0.001$ ] than non-ASOPRS males.

\section{Sex Differences in NIH Funding}

Of the 245 surgeons, 11 (4.5\%) were identified as PIs receiving NIH grants. 2.7\% (2/74) of females received NIH grants
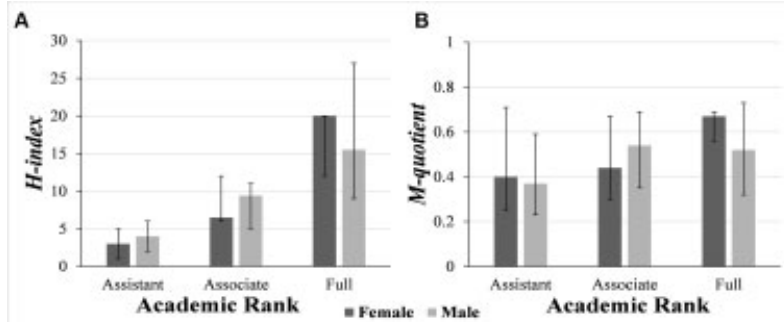

Fig. 3 Bibliometrics for oculoplastic surgeons by academic rank and sex. (A) Median h-indices. (B) Median m-quotients. Error bars representing interquartile ranges shown. No significant difference between sexes was found at each academic rank $(p>0.05)$.

compared with $5.3 \%$ (9/171) of males ( $p=0.681)$. The overall median grant awarded to female PIs was $\$ 857 \mathrm{~K}$ (IQR $\$ 16413$ ) compared with male PIs of $\$ 1.1 \mathrm{M}$ (IQR 2.0M) $(p=0.990)$. Females had a similar median number of NIH projects compared with males ( 1.5 vs. $1.0 ; p=1.000$ ).

\section{Discussion}

Previous studies have noted an under-representation of female academic ophthalmologists in senior ranked and leadership positions. ${ }^{5-8}$ For example, a study by Lopez et al. showed female ophthalmologists were under-represented at the rank of full professor. ${ }^{8}$ These findings are consistent with our results, as a smaller proportion of females were full professors and a greater proportion were assistant professors compared with males. However, our study also highlights the importance of interpreting sex differences in academic rank within the context of an individual's career duration. When career duration is considered, no difference in proportions of sexes was found at the rank of full or associate professor. Therefore, the disparities seen at each academic rank in proportion of sexes is likely due to the shorter mean career duration of female oculoplastic surgeons. As shown in - Fig. 1, only $10.8 \%$ of female surgeons have a career duration $\geq 29$ years. As more female ophthalmology residents progress through their career, differences between sexes at advanced academic ranks may diminish. Interestingly, women had a shorter career duration than men at every academic rank (assistant, associate, and full). This might suggest that women are achieving promotion (associate and full) more quickly than men; however, this is a

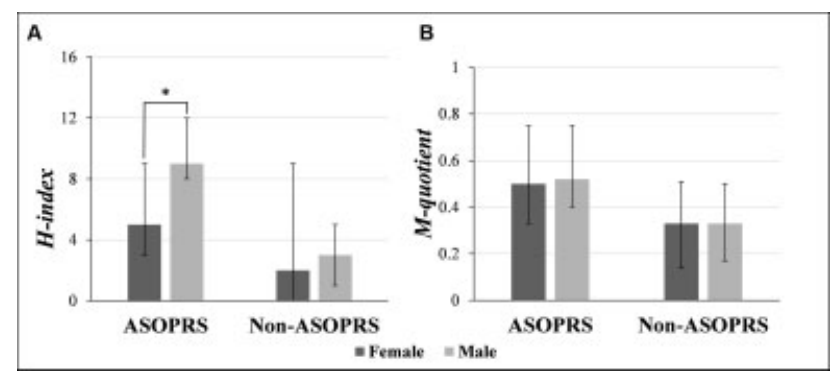

Fig. 4 Bibliometrics for oculoplastic surgeons by ASOPRS membership status and sex. (A) Median $h$-indices. (B) Median $m$-quotients. Error bars representing interquartile ranges shown. ${ }^{*} p<0.05$. 
premature conclusion as time of promotion was not readily available for analysis in this study.

Sex differences in academic rank were evaluated in the context of productivity. The $h$-index and $m$-quotient are reliable tools used to assess an individual's publication output and citation impact and readiness for promotion, and are positively associated with increasing academic rank. ${ }^{9,13}$ Thiessen et al. found that female ophthalmologists had lower $h$-indices but similar $m$-quotients compared with males. ${ }^{13}$ Our study confirms these findings within the subgroup of oculoplastic surgeons. In this study, however, the $h$-indices and $m$-quotients of females and males were also compared at different career duration intervals (-Fig. 2). Females' $h$-indices were lower early in their career ( 0 -15 years), but similar mid-career (1628 years) and late career ( $\geq 29$ years). Previous studies have shown similar early career disparities between female and male academic physicians. ${ }^{15,16}$ One possible explanation might be greater involvement in family responsibilities among women early in their careers, resulting in a decreased productivity during this time.

A study of US surgeons showed that although burnout factors were similar between sexes, women were more likely to experience work-home conflicts and commitments that may contribute negatively to advancement. ${ }^{17}$ Jolly et. al. found that women physicians with children were spending more time on parenting or domestic activities compared with their male counterparts with children. ${ }^{18}$ Other reasons to explain the disparities observed include lack of mentoring opportunities for women and increased clinical and educational responsibilities. ${ }^{19,20}$ Although our study did not investigate these other reasons for the under-representation of women in oculoplastics, it is important to understand the context that exists for external barriers that may impact women within the field of oculoplastics.

Sex differences in publication productivity were also investigated as a function of ASOPRS membership. ASOPRS, the leading national oculoplastic society, promotes scientific research among its members of board-certified oculoplastic surgeons. A recent study by Azad et al. noted a steady increase in female representation in ASOPRS since its inception in $1969 .^{21}$ While the study found that women represented $39.4 \%$ of ASOPRS members in the last decade, we found that women represented $28.3 \%$ of ASOPRS members in academia. To our knowledge, there is a lack of studies investigating differences in publication productivity among ASOPRS and non-ASOPRS members, especially in terms of sex. Our study found that although female ASOPRS surgeons had higher $h$ indices than their non-ASOPRS female counterparts, they still had lower $h$-indices than male ASOPRS surgeons. M-quotients were, however, similar between sexes among both ASOPRS and non-ASOPRS members. Once again, this suggests that sex differences in publication productivity among ASOPRS members are due to the shorter career duration of female surgeons, and not to a real difference related to sex itself. ASOPRS membership may also be associated with opportunities that promote higher scholarly output.

The NIH is the largest funder of US biomedical research and receiving NIH funding is associated with higher publica- tion productivity. ${ }^{22,23}$ A study by Svider et al concluded that female ophthalmologists received lower NIH awards than their male ophthalmologists. ${ }^{12}$ Contrary to this finding, our study found that oculoplastic surgeons do not follow the same trends as the entire field of ophthalmology. Female and male oculoplastic surgeons were equally represented as recipients of $\mathrm{NIH}$ funding $(p=0.681)$ and no difference in the median amount of $\mathrm{NIH}$ awards $(p=1.000)$ or number of projects $(p=1.000)$ were found between sexes. This is a particularly interesting statistic, since the majority of female oculoplastic surgeons are in an early stage of their career (-Fig. 1).

This study has several limitations. The accuracy of our data is dependent on the available information provided on official departmental websites and online search engines. The sex of an individual was assigned through the evaluation of photographs, pronouns, and names; however, the assigned binary sex categories may not align with how an individual may self-report their sex identification. ${ }^{24}$ While significant attempts were made to find alternative names to obtain scholarly productivity metrics and NIH funding data for the same individual, it is possible that some alternative names were not identified, which may disproportionately affect women with maiden names. Although we would have preferred to base career duration length on fellowship graduation year, residency graduation year was used instead due to its greater accessibility. Because part-time appointment status is not commonly stated on institutional websites, it is possible this study's data includes part-time faculty.

There are also inherent limitations in using databases such as Scopus and NIH RePORTER in addition to flaws with productivity indices. The SCOPUS database primarily includes only PubMed indexed articles, which may provide inaccurate publication productivity data for an individual author. $^{25}$ In addition, the NIH RePORTER database only captures NIH grants dating back to 1985 and, thus, some NIH grants may not have been identified, which may disproportionately under-report NIH awards of older investigators. The $h$-index fails to account for an author's career duration and provides an advantage to individuals later in their career who have had time to accumulate citations and publications. Thus, our current study aimed to compensate for this by analyzing the m-quotient, which may be a better measure of publication productivity when comparing groups with differing career duration, like female and male oculoplastic surgeons. In the present study, we attempted to compensate for this by also analyzing the m-quotient; however, even the $m$-quotient has inherent limitations. For example, the $m$-quotient might be artificially inflated in individuals with publications prior to the end of their residency. This would particularly impact individuals with multiple terminal degrees, who may have had a robust research portfolio prior their residency graduation. In our study, more men than women had multiple advanced degrees (PhD, MS, MPH). Although the difference was not statistically significant, this could have skewed the data to make it appear as though these individuals also had a higher m-quotient. 
Lastly, although this study highlights potential sex differences among oculoplastic surgeons, our study cannot infer causality.

\section{Conclusion}

To our knowledge, this is the first study to analyze the sex differences among academic oculoplastic surgeons in terms of academic rank, publication productivity, and NIH funding, while also investigating the association of ASOPRS membership and scholarly output. The results of our study indicated that sex differences seen in academic rank and in publication productivity are likely due to differences in career duration between the sexes. As time passes, more females will enter advanced stages of their careers and differences seen between sexes in senior academic positions and publication productivity will likely attenuate. Our study also found that ASOPRS membership may provide opportunities for increased scholarly output.

\section{Financial Disclosure}

S.M.P. serves in the role of consultant, advisory board member, and Medical Monitor for Carl Zeiss Meditec. He also serves in the role of consultant to Bausch \& Lomb. These relationships are not relevant to the present work. Remaining authors have no financial or other interests to disclose.

\section{Acknowledgments and Funding}

The work was supported by the National Center for Advancing Translational Sciences, National Institutes of Health (NIH), through Grant UL1 TR002014 and Grant UL1 TR00045. The content is solely the responsibility of the authors and does not necessarily represent the official views of the NIH, Bethesda, Maryland, United States. No other acknowledgments.

\section{References}

1 Association of American Medical Colleges. Active Physicians by Sex and Specialty 2017. Accessed April 1, 2020 at: https://www. aamc.org/data-reports/workforce/interactive-data/active-physicians-sex-and-specialty-2017

2 Christmann L. Women and their Role in Ophthalmology. Women In Ophthalmology. Accessed April 1, 2020 at: https://www. wioonline.org/news/women-role-ophthalmology/2015

3 Barzansky B, Etzel SI. Medical Schools in the United States, 20182019. JAMA 2019;322(10):986-995

4 Xierali IM, Nivet MA, Wilson MR. Current and future status of diversity in ophthalmologist workforce. JAMA Ophthalmol 2016; 134(09):1016-1023

5 Cruz OA, Johnson NB, Thomas SM. Twenty-five years of leadership: a look at trends in tenure and appointments of chairs of ophthalmology. Ophthalmology 2009;116(04):807-811

6 Camacci ML, Lu A, Lehman EB, Scott IU, Bowie E, Pantanelli SM. Association between sex composition and publication productivity of journal editorial and professional society board members in ophthalmology. JAMA Ophthalmol 2020;138(05):451-458
7 Dotan G, Qureshi HM, Gaton DD. Chairs of United States Academic Ophthalmology Departments: a descriptive analysis and trends. Am J Ophthalmol 2018;196:26-33

8 Lopez SA, Svider PF, Misra P, Bhagat N, Langer PD, Eloy JA. Gender differences in promotion and scholarly impact: an analysis of 1460 academic ophthalmologists. J Surg Educ 2014;71(06): 851-859

9 Hirsch JE. An index to quantify an individual's scientific research output. Proc Natl Acad Sci U S A 2005;102(46):16569-16572

10 Eloy JA, Svider PF, Cherla DV, et al. Gender disparities in research productivity among 9952 academic physicians. Laryngoscope 2013;123(08):1865-1875

11 Svider PF, Lopez SA, Husain Q, Bhagat N, Eloy JA, Langer PD. The association between scholarly impact and National Institutes of Health funding in ophthalmology. Ophthalmology 2014;121(01): 423-428

12 Svider PF, D'Aguillo CM, White PE, et al. Gender differences in successful National Institutes of Health funding in ophthalmology. J Surg Educ 2014;71(05):680-688

13 Thiessen C, Venable G, Ridenhour N, Kerr N. Publication productivity for academic ophthalmologists and academic ophthalmology departments in the United States: an analytical report. J Clin Acad Ophthalmol 2016;08(01):e19-e29

14 Harris PA, Taylor R, Thielke R, Payne J, Gonzalez N, Conde JG. Research electronic data capture (REDCap)-a metadata-driven methodology and workflow process for providing translational research informatics support. J Biomed Inform 2009;42(02): 377-381

15 Reed DA, Enders F, Lindor R, McClees M, Lindor KD. Gender differences in academic productivity and leadership appointments of physicians throughout academic careers. Acad Med 2011;86(01):43-47

16 Eloy JA, Svider P, Chandrasekhar SS, et al. Gender disparities in scholarly productivity within academic otolaryngology departments. Otolaryngol Head Neck Surg 2013;148(02):215-222

17 Dyrbye LN, Shanafelt TD, Balch CM, Satele D, Sloan J, Freischlag J. Relationship between work-home conflicts and burnout among American surgeons: a comparison by sex. Arch Surg 2011;146 (02):211-217

18 Jolly S, Griffith KA, DeCastro R, Stewart A, Ubel P, Jagsi R. Gender differences in time spent on parenting and domestic responsibilities by high-achieving young physician-researchers. Ann Intern Med 2014;160(05):344-353

19 Zhuge Y, Kaufman J, Simeone DM, Chen H, Velazquez OC. Is there still a glass ceiling for women in academic surgery? Ann Surg 2011;253(04):637-643

20 Kaplan SH, Sullivan LM, Dukes KA, Phillips CF, Kelch RP, Schaller JG. Sex differences in academic advancement. Results of a national study of pediatricians. N Engl J Med 1996;335(17):1282-1289

21 Azad AD, Rosenblatt TR, Chandramohan A, Fountain TR, Kossler AL. Progress towards parity. Ophthalmic Plast Reconstr Surg 2020;37(03):236-240

22 Nicholson JM, Ioannidis JP. Research grants: conform and be funded. Nature 2012;492(7427):34-36

23 Colaco M, Svider PF, Mauro KM, Eloy JA, Jackson-Rosario I. Is there a relationship between National Institutes of Health funding and research impact on academic urology? J Urol 2013;190(03): 999-1003

24 Fraser G. Evaluating inclusive gender identity measures for use in quantitative psychological research. Psychol Sex 2018;9(04): 343-357

25 Kokol P, Vošner HB. Discrepancies among Scopus, Web of Science, and PubMed coverage of funding information in medical journal articles. J Med Libr Assoc 2018;106(01):81-86 